\title{
Searching for Planets in the Hyades IV: Differential Abundance Analysis of Hyades Dwarfs ${ }^{1}$
}

\author{
Diane B. Paulson, Christopher Sneden \\ Department of Astronomy, University of Texas, Austin, TX 78712 \\ apodis@astro.as.utexas.edu, chris@verdi.as.utexas.edu \\ William D. Cochran \\ McDonald Observatory, University of Texas, Austin, TX 78712 \\ wdc@astro.as.utexas.edu
}

\begin{abstract}
We present a differential abundance analysis of Hyades F-K dwarfs in search for evidence of stellar enrichment from accreted hydrogen deficient disk material. Metallicites and relative abundance ratios of several species have been determined. We derive a cluster mean $[\mathrm{Fe} / \mathrm{H}]=$ $0.13 \pm 0.01$. Two stars show abundances $\sim 0.2$ dex larger than the cluster mean. Additionally, one star, which was added by a recent study as a cluster member, shows significantly lower abundances than the cluster mean. These three stars have questionable membership characteristics. The remaining stars in the survey have an rms of 0.04 dex in the differential $[\mathrm{Fe} / \mathrm{H}]$ values. The Hyades cluster members have apparently not been significantly chemically enriched. The abundance ratios of $\mathrm{Si}, \mathrm{Ti}, \mathrm{Na}, \mathrm{Mg}, \mathrm{Ca}$ and $\mathrm{Zn}$ with respect to $\mathrm{Fe}$ are in their solar proportions.
\end{abstract}

Subject headings: clusters: open (Hyades) — stars: abundances

\section{Introduction}

The proposition that extrasolar planet host stars tend to be metal rich has implications for the planet formation community. This fact, as shown by e.g. Gonzalez (1997, 1998), Laughlin \& Adams (1997) and Jeffery et al. (1997), although interesting, has not yet been given a single, universally accepted theoretical explanation. Two possibilities are reviewed well by Murray \& Chaboyer (2002) and Smith et al. (2001) - either planets form preferentially around stars which are intrinsically richer in heavy elements, or the overabundance of metals is due to hydrogen deficient

\footnotetext{
${ }^{1}$ Some data presented herein were obtained at the W.M. Keck Observatory, which is operated as a scientific partnership among the California Institute of Technology, the University of California and the National Aeronautics and Space Administration. The Observatory was made possible by the generous financial support of the W.M. Keck Foundation.
}

protoplanetary debris enriching the stellar photosphere. Only stars which have shallow convection regions will show metal enrichment due to accretion; when a star has a large convective region, the additional metals will be diluted beyond detection. For F stars and earlier, enrichment can occur during the early life as a main-sequence star, however, for G stars, enrichment is thought to occur only if the accretion has taken place after the first $10 \mathrm{Myr}$ of pre-main sequence evolution. At this time the convective region has decreased in size (Murray \& Chaboyer 2002; Pinsonneault et al. 2001; Laughlin \& Adams 1997). Later type stars should show no detectable enhancement, even if large amounts of material are accreted.

Current searches for planets include stars with vastly different chemical and formation histories. One good way to test the enrichment theory is by observing a star cluster, whose members were presumably formed from homogeneous material. The 
key is to look for star-to-star differences in heavy element content. Stars showing higher amounts of metals would have had to be enriched in some way, notably from $\mathrm{H}$ deficient material being accreted onto the stellar atmosphere. Similar programs have utilized this concept of eliminating initial composition variable by performing a differential abundance analysis of binary stars (Laws \& Gonzalez 2001; Gratton et al. 2001). And recently, a program similar to this one has been undertaken by Fulbright (2002).

Abundances of Hyades stars have been determined by several groups over the past few decades. These studies have provided increasingly more accurate abundances, as atomic data have improved and as stellar atmosphere models have become closer approximations to physical reality. Varenne \& Monier (1999) review the abundance studies of the Hyades from Conti et al. (1965) through their own work. It is evident that the measurement of heavy elements have been studied in A-F stars, but a detailed analysis in the lower mass dwarfs is lacking. In addition, many of these studies only include 1-2 dozen stars. Conti et al. studied various elements in 10 Hyades stars. They were also interested in looking for star-to-star differences to determine if the protocluster nebula was homogeneous. This survey provided the first evidence that $\mathrm{Li}$ in the Hyades is not uniform, while the abundances of several other elements were. To within their stated error bars, Conti et al. determined that the abundance of Hyades members is constant for all elements but Li. Later, chemical composition studies (excluding studies of only $\mathrm{Fe}$ and/or $\mathrm{Li}$, which are more numerous) were completed in A-F stars by Boesgaard et al. (1977), Burkhart \& Coupry (1989), Friel \& Boesgaard (1990), Garcia Lopez et al. (1993), Takeda \& Sadakane (1997), Hui-Bon-Hoa \& Alecian (1998), Burkhart \& Coupry (2000), and Takeda et al. (1998). For lower mass stars, heavy element abundance determinations were only completed by the following (again excluding those studies only of $\mathrm{Fe}$ and/or Li): Conti et al. (1965), King \& Hiltgen (1996) and Boesgaard et al. (1977). Papers also instrumental to the metallicity determination of the Hyades cluster are Boss (1989), Branch et al. (1980), Boesgaard \& Budge (1988), Cayrel et al. (1985), and Chaffee et al. (1971).

Pinsonneault et al. (2001) have estimated the amount of Fe that must be accreted onto the stellar photosphere in order to enrich the measured $[\mathrm{Fe} / \mathrm{H}]$ of a solar-type star appreciably. They derive that a quantity of $10 \mathrm{M}_{\oplus}$ of $\mathrm{Fe}$ (a rough upper limit to the Fe core of Jupiter) would increase the $[\mathrm{Fe} / \mathrm{H}]$ of a solar-type star by 0.09 dex. Within the errors of stellar abundance analysis and atomic data, variations of this magnitude within the cluster are detectable through a differential abundance analysis.

In this paper, one in a series exploring planets and planet formation in the Hyades cluster (e.g. Cochran et al. 2002, Paulson et al. 2002, 2003) we present abundance determinations for $\mathrm{Fe}, \mathrm{Si}, \mathrm{Ti}$, $\mathrm{Ca}, \mathrm{Na}, \mathrm{Mg}$ and $\mathrm{Zn}$ (as well as differential measurements for each of these elements) for a large sample of Hyades members over a wide effective temperature range in search for evidence of stellar enrichment.

\section{Observations}

\subsection{Sample}

The planet search program, undertaken with the Keck I HIRES (Vogt et al. 1994), contains 98 F-M dwarfs (Cochran et al. 2002). The present chemical composition analysis, which makes use of these spectra, unfortunately, does not include all 98 stars. The $\mathrm{M}$ dwarfs and a few late $\mathrm{K}$ dwarfs (B-V cutoff of 1.0) were not analyzed due to the crowded spectra, inability to accurately place the continuum, and the poorer $\mathrm{S} / \mathrm{N}$ achieved for these stars. At the beginning of the survey, we selected only stars which were thought to have no stellar companions. In a few cases, we did select wide binaries which were sufficiently separated in the sky so that we would have no contamination in the spectra from the companion. However, since that time, four stars in the original sample are now known to have non-planetary-mass companions (vB 5, vB 52, vB 17- Patience et al. (1998) and $\mathrm{vB} 88)$. The discovery of these stellar companions does not exclude us from detecting planets, nor should it affect the overall abundance determinations in this paper. Stars showing linear trends in radial velocity (perhaps additional unknown binaries) have therefore been included. Also included in this sample is one star, HD 14127, which has been monitored in the planet search program but which, we are now confident, is not a 
member. A second, HIP 13600, may also not be a member. These are discussed further in $§ 3.6$. The final sample size for this abundance study is 55 stars.

\subsection{Spectra}

A full description of the observations can be found in Cochran et al. (2002). All spectra were obtained at the Keck I telescope from 1996-2002. We have used HIRES with resolving power $(\mathrm{R}=$ $\Delta \lambda / \lambda)$ nominally at 60,000 . The signal-to-noise ratio $(\mathrm{S} / \mathrm{N})$ of each spectrum is typically $100-200$

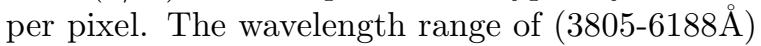
was chosen so that all $\mathrm{I}_{2}$ absorption lines are included for radial velocity measurements and so that the Ca II H\&K lines could be monitored for stellar activity. Unfortunately, this wavelength range excludes the spectral lines of many interesting elements (e.g. Li). The spectra used for abundance analysis are those taken as "template" spectra for use in the radial velocity program. Thus, these spectra are free of $\mathrm{I}_{2}$ absorption. Observations of hot stars are unnecessary due to the extreme lack of moisture at the Mauna Kea site. Therefore, the telluric absorption will be very weak. All Keck spectra were reduced using standard IRAF ${ }^{2}$ packages.

\section{Abundance Analysis}

The abundance analysis makes use of the current version of the LTE line analysis code MOOG (Sneden 1973). The linelist is compiled from three sources- Fe I and II $g f$-values were derived internally (as described in $\S 3.2$ ) and the remaining parameters were obtained from either R. E. Luck (2003, private communication) or Kurucz \& Bell (1995). The final linelist is given in Table 1, and the sources for excitation potential $(\chi)$ and oscillator strength ( $g f$-value) are listed as well. In deriving $[\mathrm{Fe} / \mathrm{H}]^{3}$ and $[\mathrm{X} / \mathrm{Fe}]$ values, solar values were derived from a solar spectrum (of Ceres) taken through HIRES. Originally, we adopted the values from Grevesse \& Sauval (1998), but we found a dif-

${ }^{2}$ IRAF is distributed by the National Optical Astronomy Observatories, which are operated by the Association of Universities for Research in Astronomy, Inc., under cooperative agreement with the National Science Foundation.

${ }^{3}[\mathrm{~A} / \mathrm{B}]=\log (\mathrm{A} / \mathrm{B})_{\star}-\log (\mathrm{A} / \mathrm{B})_{\odot}$ ference of 0.14 dex between our derived $\log \epsilon(\mathrm{Fe}) \odot^{4}$ and that found by Grevesse \& Sauval. The difference is primarily due to instrumental effects. Thus, in order to eliminate instrumental uncertainties, we used $\log \epsilon(\mathrm{Fe}) \odot$ as derived from our solar spectrum. The values of $\log \epsilon(\mathrm{X}) \odot$ were also in disagreement with Grevesse \& Sauval, so again, we chose to use the values derived from our solar spectrum.

\subsection{Stellar parameters}

We determined stellar parameters using the template spectra obtained from Keck HIRES. We normalized the continuum using IRAF. All equivalent widths (EW's) were measured by fitting a Gaussian to the observed line profiles, also using IRAF.

The stellar models used were interpolated ${ }^{5}$ atmosphere models (with no convective overshoot) based on the 1995 version of ATLAS9 code (Castelli et al. 1997). The relevant stellar parameters - effective temperature $\left(T_{\text {eff }}\right)$, gravity $(\log g)$, and microturbulence $(\xi)$, were determined in the following manner. $T_{\text {eff }}$ values were obtained by requiring that $\mathrm{Fe}$ abundances of individual lines be independent of excitation potential $(\chi)$. Microturbulence was determined by forcing Fe abundances from individual Fe I lines to be independent of line strength. Surface gravity was derived by requiring ionization equilibrium - the Fe abundance derived from Fe I lines must match that derived from Fe II lines. All stellar parameters are determined simultaneously, with the only requirement being that $\log g$ is confined to the range 4.2 to 4.7 , a reasonable range given the known cluster distance. Derived stellar parameters are listed in Table 2. The method by which we determine stellar parameters also gives us the $\log \epsilon(\mathrm{Fe})$ for each star. We thus list $[\mathrm{Fe} / \mathrm{H}]$ for each star in Table 3.

In our abundance computations, we chose a van der Waals line damping parameter option with the Unsöld (1955) approximation. We also experimented with other damping enhancements recommended by Blackwell et al. (1995) and Holweger

\footnotetext{
${ }^{4}$ where $\log \epsilon(\mathrm{Fe}) \odot=\mathrm{n}_{\mathrm{Fe}} / \mathrm{n}_{\mathrm{H}}+12.0$

${ }^{5}$ Interpolation software was kindly supplied by McWilliam (1995, private communication) and updated by Ivans (2002, private communication).
} 
(1971) to determine if one is significantly better for this set of data than the others. All three damping options were tested using both the curve of growth analysis and by comparing the lineshapes in a spectral region using a synthesis approach. We determined that neither of these enhancements to the Unsöld approximation yields a better fit to the lines chosen. The effect of damping was more apparent in the abundance analysis, where it was clear that the damping parameter affected the cooler stars more than the warmer stars (yielding slightly different abundances along the mainsequence). In an absolute abundance analysis, the choice of different damping parameters does not seem to affect the final results. In an absolute abundance analysis, the comparison is made to the overall solar abundance, which changes only slightly with the different damping options (0.02 dex). The effect of damping on a differential analysis becomes slightly more pronounced. We compared the results of differential abundances of two stars and the sun with varying damping parameters. The greatest difference was in vB 143 (compared to the standard vB 153) which showed changes of 0.06 dex between the Holweger and the Blackwell et al. suggested enhancements. The solar spectrum (also compared to vB 153) showed a difference of 0.04 dex and vB 15 (compared to vB 153) showed 0.03 dex. There is no significant trend in these results with stellar temperature, indicating that the choice of damping should not adversely affect the differential analysis. We note that Prochaska et al. (2000) also see inconsistencies between these damping enhancements.

The choice of models with no convective overshoot was made both by taking into consideration the recommendations of Castelli et al. (1997) and by empirical testing. We first used models with convective overshoot, but we found a significant linear trend of increasing $[\mathrm{Fe} / \mathrm{H}]$ with increasing $T_{\text {eff }}$. Initially we thought we were seeing the effects of uniform enrichment up the main sequence. However the majority of our program stars are $\mathrm{G}$ and $\mathrm{K}$ dwarfs. Thus, if enrichment were uniform, there ought to be a plateau in the $K$ and late $G$ dwarfs with slight increase in early $G$ and late $F$ dwarfs. And, this is not what we were seeing. The other concern was that the slope was large (roughly 0.15 dex from $\mathrm{F}$ to $\mathrm{K}$ ). So, we experimented with models with no convective overshoot.
The trend of abundance with $T_{\text {eff }}$ disappeared by using these models. Thus, we decided to use models with no overshoot for the entire analysis.

For each star, we measured the EW's of 12-20 (presumably) unblended Fe I lines and 5-9 un-

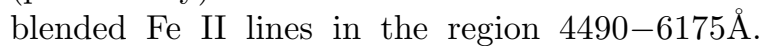
We preferentially chose lines redward of $4500 \AA$ due to the extremely crowded spectra blueward of this cutoff. Where possible we tried to maintain only lines which were significantly redward of this. Continuum placement becomes difficult in the blue end of the spectrum. The number of lines available varied according to the temperature of the star. Because the emphasis of this work is differential, absolute accuracy of elemental $g f$-values are of less importance. However, in order to obtain correct stellar parameters, we wanted to use accurate Fe $g f$-values. In the same way all EW's were measured, we varied the $g f$-values until the line abundances matched the solar model and solar abundances. We used the Kurucz solar atlas (Kurucz et al. 1984) for this analysis.

In December of 2001, C. Allende Prieto obtained spectra of several super-solar mass Hyades stars with McDonald Observatory's $2.7 \mathrm{~m}$ Harlan Smith telescope using the coudé echelle spectrograph $(\mathrm{R}=60,000$ with $\mathrm{S} / \mathrm{N}$ of about $300-500$ per pixel at $5800 \AA$ ). Of these stars, 11 were also observed in our sample at Keck, providing a comparison and check of measurements of stellar parameters between the data sets. The stars observed at McDonald are typically higher mass, and thus higher $v \sin i$ than the stars chosen for planet search at Keck. So, several of the stars observed by C. Allende Prieto are not easily analyzed with the equivalent width method for determining abundances using the chosen linelist. We do not want to compromise the internal consistency of this analysis by adopting a separate linelist for this additional set of data. Therefore, in this paper, we will only discuss the analysis of the 11 stars common to both data sets.

We compare the stellar parameters derived for these 11 stars in Table 4. EW's measured from the McDonald spectra tend to be, on average, a few $m \AA$ larger than the Keck spectra. This yields slightly higher (roughly 0.05 dex) abundances for $[\mathrm{Fe} / \mathrm{H}]$. This enhancement is completely due to the use of different spectrographs. In general, the stellar parameters we derive are consistent between 
the 2 data sets.

Our determination of $T_{\text {eff }}$ also agrees well with the $T_{\text {eff }}$ derived for stars in common with Allende Prieto \& Lambert (1999) as shown in Figure 3, noting that Allende Prieto \& Lambert list $\log T_{\text {eff }}$ rounded to 2 decimal places. Thus, in Figure 3 , the converted $T_{\text {eff }}$ from Allende Prieto \& Lambert values appear to be in discrete units. The mean difference is $\left\langle T_{\text {eff, thisstudy }}-\right.$ $\left.T_{\text {eff,Allende-Prieto }}\right\rangle=34.6 \mathrm{~K}$ with a standard deviation $\sigma=67.9 \mathrm{~K}$.

\section{2. $[\mathrm{Fe} / \mathrm{H}]$ and $[\mathrm{X} / \mathrm{Fe}]$}

Absolute $[\mathrm{Fe} / \mathrm{H}]$ abundances are derived during the process of determining stellar parameters. Using the derived stellar atmosphere models and measured EW's of various atomic lines listed in Table 1, we determined elemental abundances $([\mathrm{X} / \mathrm{Fe}])$. Derived $[\mathrm{Fe} / \mathrm{H}]$ and $[\mathrm{X} / \mathrm{Fe}]$ values are listed for each star in Table 3.

Our derived mean Hyades metallicity is $\langle[\mathrm{Fe} / \mathrm{H}]\rangle$ $=0.13 \pm 0.01$ with $\sigma=0.05$, not including HD 14127 . If we also exclude $\mathrm{vB} 1$ and $\mathrm{vB} 2$, we obtain $\langle[\mathrm{Fe} / \mathrm{H}]\rangle=0.13 \pm 0.01, \sigma=0.04$. This is in agreement with various surveys of Hyades metallicity, e.g. $\langle[\mathrm{Fe} / \mathrm{H}]\rangle=0.16 \pm 0.04$ (Boesgaard et al. 2002), $0.20 \pm 0.10$ (Branch et al. 1980), 0.130 \pm 0.026 (Boss 1989), and $0.12 \pm 0.03$ (Cayrel et al. 1985).

We now consider the stars with $[\mathrm{Fe} / \mathrm{H}]$ not within $1 \sigma$ of the cluster mean (these outliers can be seen in the differential $[\mathrm{Fe} / \mathrm{H}]$ plot of Figure 2 , which is further discussed in §3.3). The two outliers with $\Delta[\mathrm{Fe} / \mathrm{H}]$ (and $[\mathrm{Fe} / \mathrm{H}]$ ) lower than the cluster mean (dashed line), HD 14127 and HIP 13600, are disregarded due to questionable membership, as discussed in $\$ 3.6$.

One might assume that for the outliers with high $[\mathrm{Fe} / \mathrm{H}]$ (and $\Delta[\mathrm{Fe} / \mathrm{H}])(\mathrm{vB} 19$, vB 1 and vB 2, as seen in Figure 2) we have determined an incorrect set of model parameters - that, perhaps, our determined $T_{\text {eff }}$ are too high. However, changing the model parameters by reasonable amounts cannot solve the entire problem of their high abundances. For vB 19, the determination of $\log g$ may be questionable due to the fact that we were only able to measure $4 \mathrm{Fe}$ II lines in the spectrum. If the gravity is questionable, then the other parameters may be off slightly as well. We have derived an $T_{\text {eff }}$ which is roughly $150 \mathrm{~K}$ higher than Allende
Prieto \& Lambert (1999) (hereafter APL99) derived. We note that APL99 interpolate theoretical isochrones with observed data from HIPPARCOS to get stellar parameters, which is a different approach than what we use. Thus, some amount of disagreement is understandable between our study and theirs. To test our abundance determination, though, we force vB 19 to have APL99's derived temperature and rederive the other stellar parameters. We find $\xi=1.0 \mathrm{~km} \mathrm{~s}^{-1}$ and $\log g=3.9$. Together, these new parameters give an $[\mathrm{Fe} / \mathrm{H}]$ of 0.16 . This is within $1 \sigma$ of the cluster mean, though still on the high end. However, the new gravity derived is in strong disagreement with 4.40 as derived by APL99. So, assuming that the disagreement in $\log g$ can be explained by EW measurement error, the high abundance in vB 19 may be reduced to within $1 \sigma$ of the cluster mean. Thus, we do not feel strongly that vB 19 is, indeed, enhanced relative to the cluster.

Doing the same test for $\mathrm{vB} 1$, forcing a $T_{\text {eff }}$ of $6165 \mathrm{~K}$ gives $\log g$ of 4.1 (in disagreement with APL99's 4.51), $\xi$ of $0.8 \mathrm{~km} \mathrm{~s}^{-1}$, and [Fe/H] of 0.23 , still significantly higher than the cluster mean. For the final case of vB 2, forcing the $T_{\text {eff }}$ to be 5888 $\mathrm{K}$ gives $\log g$ of 4.1 (APL99 derived 4.40), $\xi$ of $0.6 \mathrm{~km} \mathrm{~s}^{-1}$, and $[\mathrm{Fe} / \mathrm{H}]$ of 0.24 (also significantly higher than the cluster mean). So, the high abundances of $\mathrm{vB} 1$ and $\mathrm{vB} 2$ cannot simply be explained by poor choice of models unless the true model parameters are drastically inconsistent with what we have measured. Additionally, in his initial analysis, Fulbright (2002) also sees an enrichment in these two stars. So, either vB 1 and vB 2 are not members or they have been enriched relative to the cluster mean. The membership of these stars is further discussed in $§ 3.6$.

\subsection{Differential $[\mathrm{Fe} / \mathrm{H}]$ and $[\mathrm{X} / \mathrm{Fe}]$}

By employing a differential abundance analysis (e.g. Gray 1992), one removes the uncertainty in the oscillator strengths of the lines, which are often poorly known. Therefore, to answer the question of whether we see enrichment of metals within the Hyades cluster, we use a self-consistent, differential abundance analysis to look for any starto-star metallicity variations. We do not employ the method of also deriving differential stellar parameters as Laws \& Gonzalez (2001) do, because our $T_{\text {eff }}$ range is too large. In doing a line-by-line 
differential analysis, we will be able to place upper limits on the amount of $\mathrm{H}$ deficient debris that could have been accreted onto a star's photosphere relative to the cluster mean.

In order to get differential abundances $(\Delta[\mathrm{Fe} / \mathrm{H}]$ and $\Delta[\mathrm{X} / \mathrm{Fe}])$ we subtract $\log \epsilon(\mathrm{X})$ of each line in each star with the same line in a comparison star (we chose the $\mathrm{K}$ dwarf vB 153). Thus, $\Delta[\mathrm{Fe} / \mathrm{H}]$ and $\Delta[\mathrm{X} / \mathrm{Fe}]$ are the means of the differences for all lines in a given star. The scatter is significantly reduced. This gives a more accurate relative abundance than we can obtain by just taking the mean of all lines and subtracting that value from solar (the values $[\mathrm{Fe} / \mathrm{H}]$ and $[\mathrm{X} / \mathrm{Fe}]$ ). Differential abundance values are listed in Table 5 and are plotted in Figures 2 and 4 for each species, with typical error bars shown in the bottom right hand corner of each panel. For a given element, we determine the star-to-star variations with a standard deviation about the mean $\sigma \leq 0.05$ dex and the standard deviation of the mean significantly lower (less than 0.01 dex). $\Delta[\mathrm{Si} / \mathrm{Fe}], \Delta[\mathrm{Ti} / \mathrm{Fe}]$, $\Delta[\mathrm{Na} / \mathrm{Fe}], \Delta[\mathrm{Mg} / \mathrm{Fe}]$ and $\Delta[\mathrm{Zn} / \mathrm{Fe}]$ are all fairly consistent with $\Delta[\mathrm{Fe} / \mathrm{H}]$ - they are constant along the main-sequence with small scatter. The linear least squares fits to these data reveal the following relationships:

$$
\begin{aligned}
& \Delta[\mathrm{Si} / \mathrm{Fe}]=-0.106+0.109(\mathrm{~B}-\mathrm{V}), \\
& \Delta[\mathrm{Ti} / \mathrm{Fe}]=-0.014-0.040(\mathrm{~B}-\mathrm{V}), \\
& \Delta[\mathrm{Na} / \mathrm{Fe}]=0.087-0.129(\mathrm{~B}-\mathrm{V}), \\
& \Delta[\mathrm{Mg} / \mathrm{Fe}]=-0.025-0.004(\mathrm{~B}-\mathrm{V}) \text { and } \\
& \Delta[\mathrm{Zn} / \mathrm{Fe}]=0.001-0.067(\mathrm{~B}-\mathrm{V}) .
\end{aligned}
$$

$\Delta[\mathrm{Ca} / \mathrm{Fe}]$ has a significant trend in abundance with color. The value of $\Delta[\mathrm{Ca} / \mathrm{Fe}]$ is zero at the same B-V as the comparison star. Therefore, we believe this trend is primarily due to the fact that these lines begin to move off the linear part of the curve of growth (start becoming saturated) in cooler stars. For the other elements, when a line had this type of behavior, we removed it from the list (we preferred to maintain one linelist for the entire sample of stars). However, there was not a reasonable number of lines available for us to remove all lines of $\mathrm{Ca}$ which behave like this. The least squares fit for $\mathrm{Ca}$ is

$$
\Delta[\mathrm{Ca} / \mathrm{Fe}]=0.274-0.372(\mathrm{~B}-\mathrm{V}) .
$$

In Figure 4, there are 2 severe outliers. In the $\Delta[\mathrm{Na} / \mathrm{Fe}]$ plot, vB 26 and $\mathrm{vB} 105$ have extremely high $\Delta[\mathrm{Na} / \mathrm{Fe}]$ compared to the cluster. The cause of this enhancement is saved for future study.
Finally, Table 6 lists cluster mean abundances for both absolute and differential analyses.

\section{4. $v \sin i$}

We also determine the rotational velocity $(v \sin i)$ for all stars. $v \sin i$, the instrumental profile (IP), the macroturbulence $(\zeta)$, and the limb darkening are combined to form a "smoothing" parameter. This smoothing parameter is convolved with an intensity profile for the star. Because we derive an intensity profile from the stellar models, in order to determine $v \sin i$, we only need to determine these other broadening parameters. We synthesized 5 Fe I lines in this $6150-6180 \AA$ region. For each line, we know the abundance from determination of the stellar parameters. We then fit a "smoothing" parameter to each line including calculated, measured or estimated values for each of the other broadening parameters. The IP is measured by fitting a Gaussian to the lines of the Thorium-Argon (ThAr) calibration lamp. The FWHM, as measured from the ThAr calibration spectra, varies from 0.0918 to $0.0921 \AA$ from the redmost to the bluemost lines in the chosen region. The synthesis code is insensitive to this small a change. So, we used $0.09 \AA$ as the IP broadening. We estimated $\zeta$ according to the Saar \& Osten (1997) estimates for active stars and using B-V from APL99. The limb darkening coefficient is estimated from Gray (1992). Using the individual abundances and the above smoothing parameters, the only unknown left is $v \sin i$. We took a mean of the $v \sin i$ derived for each of the 5 lines to determine the overall $v \sin i$ of the star. In this manner, we are able to determine $v \sin i$ to about $0.7 \mathrm{~km} \mathrm{~s}^{-1}$.

The upper panel of Figure 5 shows $v \sin i$ versus $\mathrm{B}-\mathrm{V}$ for our target stars, and individual measurements of $v \sin i$ are listed in Table 2 . We see the expected decrease of $v \sin i$ with decreasing mass and the expected spread due to the $\sin i$ ambiguity. To estimate the actual rotational velocity of a star in the cluster based solely on color (or mass or $T_{\text {eff }}$ ) we only need to fit a function to the upper envelope of the $v \sin i$ data. In Figure 5 we have done so for 3 different functions for our data alone. The best fit for our data was with a 5th order polynomial (solid curve). The second panel in Figure 5 shows our $v \sin i$ measurements and fits to our data along with $v \sin i$ from Böhm-Vitense et al. (2002), Benz et al. (1984) (with B-V values from SIM- 
$\mathrm{BAD}^{6}$ ), and selected dwarfs from Kraft (1965). The $v \sin i$ values taken from literature were measured in different ways and typically they do not remove the, albeit small, contribution of macroturbulence. Our fits (solid, dashed and dotted lines) do not take into account the $v \sin i$ values from literature. These fits are extended to show that, while consistent with our data, they do not correctly quantify the true rotational velocity "upper envelope" for all Hyades stars.

\subsection{Errors}

There are several sources of error when measuring stellar abundances, especially if one is interested in absolute abundances. External errors such as uncertainties in atomic parameters, choice of model atmospheres and solar abundances, can cause significant errors in determined absolute abundances, while these are minimized in differential abundance analysis. Internal errors, such as measurement of stellar absorption lines, continuum placement, and choice of stellar model parameters can be minimized and quantified to some degree. Typically, we can repeatably measure EW's to $\lesssim 1 \mathrm{~m} \AA$. On average, an overestimation of a single line's EW by $1 \mathrm{~m} \AA$ will give a higher line abundance by 0.02 dex. Individual cases obviously will depend on the $\mathrm{S} / \mathrm{N}$ of the spectra (i.e. spectra with higher levels of noise will have larger errors in EW determination). Lines having noise that caused problems in line fitting (i.e. where a noise spike was present in the line or where the feature was difficult to discern from the noise in the continuum) were not included in the analysis. Continuum placement is also a (somewhat) unquantifiable source of error. However, as an example, we changed the order of the cubic spline fit to the continuum for a given spectrum and measured the EW from the same set of lines. A change from a third order to a fifth order spline gave a decrease in the EW of, on average, $0.4 \mathrm{~m} \AA$. Additionally, we can quantify some internal errors, even though most of the fitting for the parameters $\left(T_{\mathrm{eff}}, \xi\right.$, and $\log g$ ) are done by eye. Table 7 shows the abundance dependencies on model parameters. For Mg abundance determination, we were only able to measure 1 stellar line, and for $\mathrm{Zn}$ and $\mathrm{Na}, 2$, and

\footnotetext{
${ }^{6}$ This research has made use of the SIMBAD database, operated at CDS, Strasbourg, France.
}

$\mathrm{Ca}$, only 3 . The absolute abundances quoted here for these elements are much less certain than, say, $\mathrm{Fe}$, which has significantly many more lines. For absolute $[\mathrm{Fe} / \mathrm{H}],[\mathrm{Ti} / \mathrm{H}]$ and $[\mathrm{Si} / \mathrm{H}]$, the total error on an individual measurement is about 0.1 dex, which includes both internal and external errors. Differentially, the uncertainties are much smaller, on the order of 0.05 dex. This is smaller due to the removal of certain external errors like the atomic gf parameter.

\subsection{Notes on cluster membership}

The following 9 stars have at least one anomalous characteristic compared to other cluster members. The stellar characteristics of interest here are metallicity, chromospheric activity level, photometry, proper motion and parallax.

HD 14127 was included as a member by (Perryman et al. 1998) (hereafter Pe98) based on HIPPARCOS observations. D. Latham (private communication, 1999) concluded that this star is not a member because it has too high a Hipparcos distance and the photometry is below the main sequence. In Paulson et al. (2002) (hereafter Pa02), we showed that that this star does have activity levels consistent with the age of the Hyades. However, this star's metallicity is 0.25 dex below the cluster mean. Thus, it is severely inconsistent with the Hyades. It is our belief that this star is not a member of the Hyades.

HIP 13600 has slightly low abundances in all elements but $\mathrm{Mg}$. In the activity analysis, it was also an outlier, showing much lower activity levels than expected for a Hyades member (Pa02). Again, this star was included by Pe98 but Latham concludes that the photometry is below the main sequence and the Hipparcos distance is too high. Hoogerwerf \& Aguilar (1999) reject this star as a cluster member. Thus, we consider HIP 13600 is a probable non-member.

vB 118, HD 26257, HD 35768, and HD 19902 all show low activity levels (Pa02), but in this analysis, they all have consistent metallicities with the Hyades cluster mean. Of these, Latham concludes that HD 26257, HD 35768 and HD 19902 are not members based on the same criterion as above. He agrees that vB 118 is a member. Pe98 includes all of these stars as members. HD 26257 and HD 35768 were rejected by Hoogerwerf \& 
Aguilar and HD 35768 was also rejected by de Bruijne et al. (2001). At this point, we still consider vB 118 to be a member. The others are considered to be probable non-members.

vB 12, also showing consistent abundances and photometry, radial velocity and Hipparcos distances, shows slightly high activity levels. We believe that this star is most likely a cluster member despite its anomalous activity level.

vB 1 and vB 2 are the two stars in the sample which have significantly higher abundances than the cluster mean. They both have consistent photometry, radial velocity and Hipparcos distances for membership. vB 1 has a slightly low activity level as compared to the cluster mean. de Bruijne et al. (2001) find that these stars are nonmembers based on the proper motion and trigonometric parallax analyses of both de Bruijne (1999) and Hoogerwerf \& Aguilar (1999). The radial velocities of both of these stars is $31 \mathrm{~km} \mathrm{~s}^{-1}$, well within the range of the cluster $\left(28-42 \mathrm{~km} \mathrm{~s}^{-1}\right)$. They have similar proper motions and are near one another in the cluster (the difference in right ascension is $6.39^{s}$ and only $2^{m} 3.6^{s}$ in declination). Our differential radial velocity curves of these two stars (which, admittedly, only spans $\sim 5$ years) do not reveal any linear trends suggesting a relationship between them; although, the possibility still remains that they are or once were a wide binary pair. Additionally, it is well known that nearby solar-type stars are generally of solar metallicity or lower, for example see the recent survey by Gaidos \& Gonzalez (2002). So, it is unlikely that these stars happen to have similar super-solar metallicities, are quite close in proximity, and are just random field stars. We consider these stars to have questionable membership, but at this time we are not able to make a more solid classification.

\section{Discussion}

It is apparent now that at the $1 \sigma$ (or $0.04 \mathrm{dex}$ ) level, most Hyades members are uniform in composition. The abundances of several elements were measured to support this assertion. The implication of this result is that because the Hyades members were formed from homogeneous material, if significant enrichment of photospheres occurred, we would be able to see evidence of the enrichment. And, since we do not see variations in mea- sured abundances, significant enrichment has not occurred in these stars. Recalling the calculations of Pinsonneault et al. (2001), enrichment of $10 \mathrm{M}_{\oplus}$ of Fe will increase the stellar $[\mathrm{Fe} / \mathrm{H}]$ by 0.09 dex. We are able to rule out enrichment of this magnitude in our higher mass stars. We are able to scale this relation and determine that we still do not see enrichment at even the $7 \mathrm{M}_{\oplus}$ level.

vB 1 and vB 2 are interesting stars. They are significantly enriched relative to the cluster mean. Early surveys have always included them as members, but recently, de Bruijne et al. (2001) assert that they are non-members. The question remains, then, if the stars are enriched members or whether they are interlopers. These two stars certainly merit significant further study.

When the search for planets concludes, we will be able to say more firmly whether planets exist in the Hyades and if they have migrated, we will be able to place firm upper limits on the amount of debris that could have been cast onto the star during this process. For now, we are only able to place upper limits on the possible enrichment due to possible disks. Moreover, we are confident that the material that formed member stars was, in fact, homogeneous.

We would like to thank Carlos Allende Prieto and David Yong for use of data obtained at McDonald Observatory. Additionally, DBP would like to thank Jennifer Simmerer for many useful discussions about stellar abundance analysis. This material is based upon work supported by the National Aeronautics and Space Administration under grant NAG5-9227 issued through the Office of Space Science. CS is supported by NSF grant AST-9987162.

\section{REFERENCES}

Allende Prieto, C. \& Lambert, D. L. 1999, A\&A, 352,555

Böhm-Vitense, E., Robinson, R., Carpenter, K., \& Mena-Werth, J. 2002, ApJ, 569, 941

Benz, W., Mayor, M., \& Mermilliod, J. C. 1984, A\&A, 138, 93

Blackwell, D. E., Lynas-Gray, A. E., \& Smith, G. 1995, A\&A, 296, 217 
Boesgaard, A. M., Beard, J. L., \& King, J. R. 2002, BAAS, 34, 1170

Boesgaard, A. M. \& Budge, K. G. 1988, ApJ, 332, 410

Boesgaard, A. M., Heacox, W. D., \& Conti, P. S. 1977, ApJ, 214, 124

Boss, A. P. 1989, PASP, 101, 767

Branch, D., Lambert, D. L., \& Tomkin, J. 1980, ApJ, 241, L83

Burkhart, C. \& Coupry, M. F. 1989, A\&A, 220, 197

—. 2000, A\&A, 354, 216

Castelli, F., Gratton, R. G., \& Kurucz, R. L. 1997, A\&A, 318, 841

Cayrel, R., Cayrel de Strobel, G., \& Campbell, B. 1985, A\&A, 145, 249

Chaffee, F. H., Carbon, D. F., \& Strom, S. E. 1971, ApJ, 166, 593

Cochran, W. D., Hatzes, A. P., \& Paulson, D. B. 2002, AJ, 124, 565

Conti, P. S., Wallerstein, G., \& Wing, R. F. 1965, ApJ, 142, 999

de Bruijne, J. H. J. 1999, MNRAS, 306, 381

de Bruijne, J. H. J., Hoogerwerf, R., \& de Zeeuw, P. T. 2001, A\&A, 367, 111

Friel, E. D. \& Boesgaard, A. M. 1990, ApJ, 351, 480

Fulbright, J. P. 2002, BAAS, 34, 1128

Gaidos, E. J. \& Gonzalez, G. 2002, New Astronomy, 7,211

Garcia Lopez, R. J., Rebolo, R., Herrero, A., \& Beckman, J. E. 1993, ApJ, 412, 173

Gonzalez, G. 1997, MNRAS, 285, 403

-. 1998, A\&A, 334, 221

Gratton, R. G., Bonanno, G., Claudi, R. U., Cosentino, R., Desidera, S., Lucatello, S., \& Scuderi, S. 2001, A\&A, 377, 123
Gray, D. F. 1992, The Observation and Analysis of Stellar Photospheres (Cambridge: Cambridge University Press)

Grevesse, N. \& Sauval, A. J. 1998, Space Science Reviews, 85, 161

Holweger, H. 1971, A\&A, 10, 128

Hoogerwerf, R. \& Aguilar, L. A. 1999, MNRAS, 306,394

Hui-Bon-Hoa, A. \& Alecian, G. 1998, A\&A, 332, 224

Jeffery, C. S., Bailey, M. E., \& Chambers, J. E. 1997, The Observatory, 117, 224

King, J. R. \& Hiltgen, D. D. 1996, AJ, 112, 2650

Kraft, R. P. 1965, ApJ, 142, 681

Kurucz, R. \& Bell, B. 1995, Atomic Line Data (R.L. Kurucz and B. Bell) Kurucz CD-ROM No. 23. Cambridge, Mass.: Smithsonian Astrophysical Observatory, 1995., 23

Kurucz, R. L., Furenlid, I., \& Brault, J. 1984, Solar flux atlas from 296 to 1300 NM (National Solar Observatory Atlas, Sunspot, New Mexico: National Solar Observatory, 1984)

Laughlin, G. \& Adams, F. C. 1997, ApJ, 491, L51

Laws, C. \& Gonzalez, G. 2001, ApJ, 553, 405

Murray, N. \& Chaboyer, B. 2002, ApJ, 566, 442

Patience, J., Ghez, A. M., Reid, I. N., Weinberger, A. J., \& Matthews, K. 1998, AJ, 115, 1972

Paulson, D. B., Saar, S. H., \& Cochran, W. D. 2003, AJ, submitted

Paulson, D. B., Saar, S. H., Cochran, W. D., \& Hatzes, A. P. 2002, AJ, 124, 572

Perryman, M. A. C., Brown, A. G. A., Lebreton, Y., Gómez, A., Turon, C., Cayrel de Strobel, G., Mermilliod, J. C., Robichon, N., Kovalevsky, J., \& Crifo, F. 1998, A\&A, 331, 81

Pinsonneault, M. H., DePoy, D. L., \& Coffee, M. 2001, ApJ, 556

Prochaska, J. X., Naumov, S. O., Carney, B. W., McWilliam, A., \& Wolfe, A. M. 2000, AJ, 120, 2513 
Saar, S. \& Osten, R. 1997, MNRAS, 284, 284

Smith, V. V., Cunha, K., \& Lazzaro, D. 2001, AJ, 121,3207

Sneden, C. A. 1973, Ph.D. Thesis

Takeda, Y., Kawanomoto, S., Takada-Hidai, M., \& Sadakane, K. 1998, PASJ, 50, 509

Takeda, Y. \& Sadakane, K. 1997, PASJ, 49, 367

Unsöld, A. 1955, Physik der Sternatmospharen, MIT besonderer Berucksichtigung der Sonne. (Berlin, Springer, 1955. 2. Aufl.)

Varenne, O. \& Monier, R. 1999, A\&A, 351, 247

Vogt, S. S., Allen, S. L., Bigelow, B. C., Bresee, L., Brown, B., Cantrall, T., Conrad, A., Couture, M., Delaney, C., Epps, H. W., Hilyard, D., Hilyard, D. F., Horn, E., Jern, N., Kanto, D., Keane, M. J., Kibrick, R. I., Lewis, J. W., Osborne, J., Pardeilhan, G. H., Pfister, T., Ricketts, T., Robinson, L. B., Stover, R. J., Tucker, D., Ward, J., \& Wei, M. Z. 1994, Proc. Soc. Photo-opt. Inst. Eng., 2198, 362

This 2-column preprint was prepared with the AAS LATEX macros v5.0. 


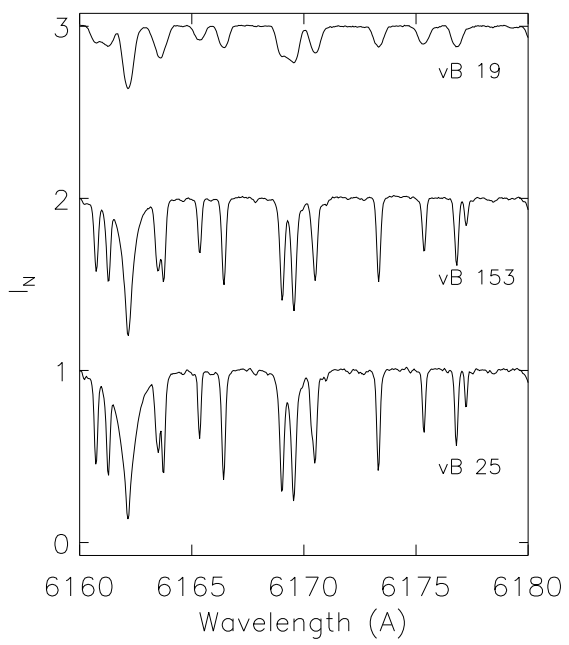

Fig. 1.- Example spectra from our survey. We show here how some lines become blended with increasing rotational velocity and increasing $T_{\text {eff }}$. We have added 1 and 2 units, respectively, to the normalized intensity $\left(\mathrm{I}_{N}\right)$ of $\mathrm{vB} 153$ and $\mathrm{vB} 19$. $\mathrm{vB} 19$ is our warmest star, vB 25 is our coolest star and vB 153 is our comparison star.

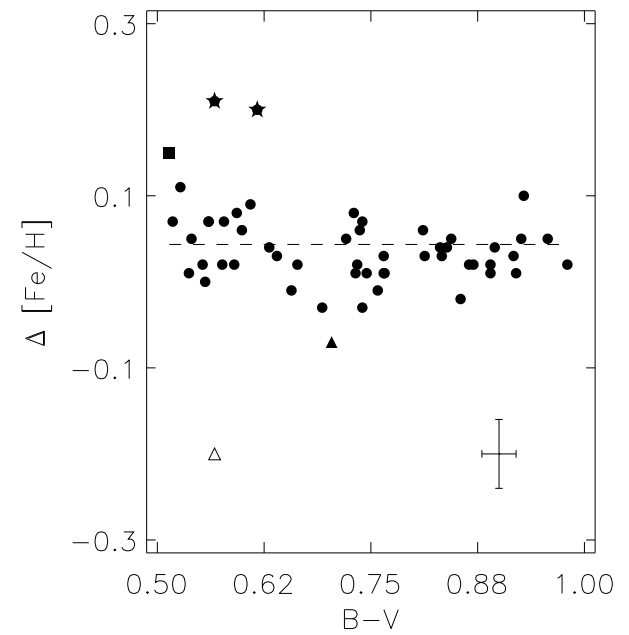

Fig. 2.-Differential $[\mathrm{Fe} / \mathrm{H}]$ of sample stars versus $\mathrm{B}-\mathrm{V}$. The differential comparison star is $\mathrm{vB} 153$, as discussed in the text. Note that the 1 very low outlier is HD 14127 (open triangle) and HIP 13600 (filled triangle) is just slightly lower in $[\mathrm{Fe} / \mathrm{H}]$ than the other members. vB 1, vB 2 (filled stars) and vB 19 (filled square) all are higher than other members. See $\S 3.6$ for membership information. The dashed line is the mean abundance level. A set of typical error bars for each measurement is shown in the bottom right hand corner of the plot.

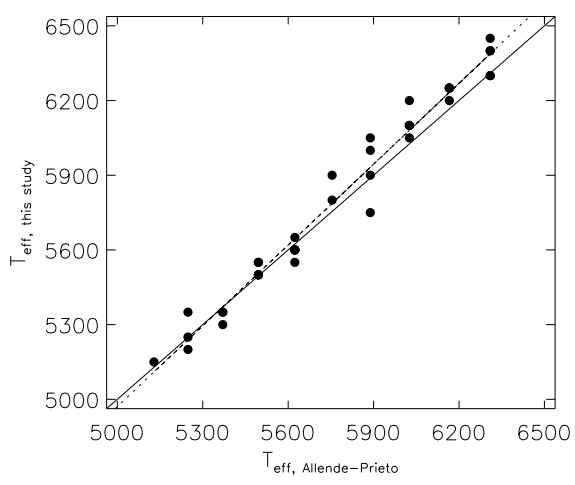

Fig. 3. - A comparison of $T_{\text {eff }}$ derived in this study versus those derived by Allende Prieto \& Lambert (1999). The solid line is 1:1, and the dashed line is a least squares fit to the data. 

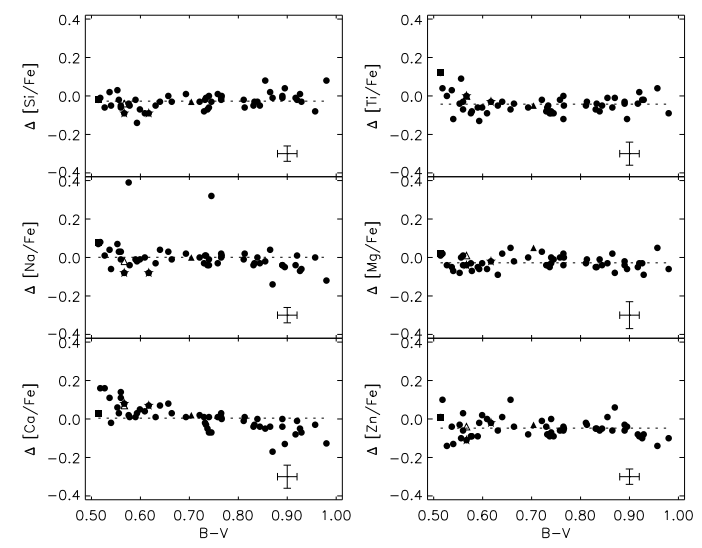

Fig. 4.-Differential $[\mathrm{X} / \mathrm{Fe}]$ of sample stars versus $\mathrm{B}-\mathrm{V}$. The differential comparison star is $\mathrm{vB} 153$, as discussed in the text. The symbols are the same as discussed in Figure 2. Dashed lines are mean abundance levels. Typical error bars are shown in each panel in the bottom right corner.

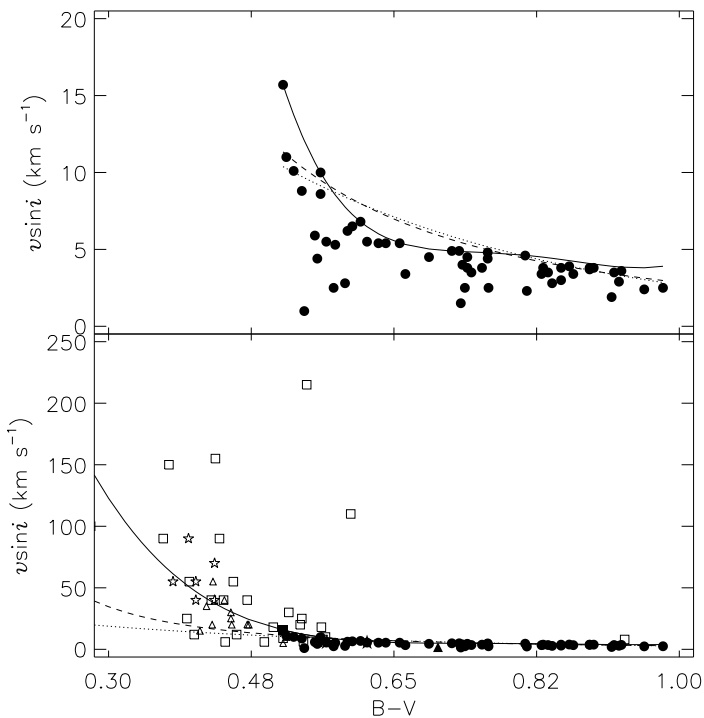

Fig. 5.- $v \sin i$ of observed Hyades stars (including the same possible non-members as discussed in Figure 2). The symbols are the same as in Figure 2. The solid curve is a polynomial fit to the upper envelope of our data (filled circles) as discussed in the text, the dashed curve is a power law to our data only and the dotted curve is an exponential fit to our data, as well. The upper panel shows only our data whereas the bottom panel adds in $v \sin i$ measurements from Böhm-Vitense et al. (2002) (open triangles), Benz et al. (1984) (open stars), and selected dwarfs from Kraft (1965) (open squares). 
TABLE 1

LINE LIST

\begin{tabular}{|c|c|c|c|c|}
\hline Species & Wavelength & $\chi(\mathrm{eV})$ & $\log g f$ & Reference \\
\hline $\mathrm{Na} \mathrm{I}$ & 6154.226 & 2.100 & -1.570 & 1 \\
\hline $\mathrm{Na} \mathrm{I}$ & 6160.747 & 2.100 & -1.270 & 1 \\
\hline $\mathrm{Mg} \mathrm{I}$ & 5711.088 & 4.346 & -1.833 & 2 \\
\hline Si I & 5948.541 & 5.080 & -1.230 & 1 \\
\hline Si I & 6125.021 & 5.610 & -1.513 & 1 \\
\hline Si I & 6142.483 & 5.620 & -1.540 & 1 \\
\hline Si I & 6145.016 & 5.610 & -1.479 & 1 \\
\hline $\mathrm{Ca} \mathrm{I}$ & 5260.387 & 2.521 & -1.719 & 1 \\
\hline $\mathrm{Ca} \mathrm{I}$ & 6166.439 & 2.520 & -1.142 & 1 \\
\hline $\mathrm{Ca} \mathrm{I}$ & 6169.042 & 2.520 & -0.797 & 1 \\
\hline $\mathrm{Ti} \mathrm{I}$ & 5922.110 & 1.046 & -1.410 & 1 \\
\hline $\mathrm{Ti} \mathrm{I}$ & 5937.811 & 1.066 & -1.834 & 1 \\
\hline Ti I & 5941.752 & 1.053 & -1.454 & 1 \\
\hline Ti I & 5953.162 & 1.887 & -0.273 & 1 \\
\hline Ti I & 5965.828 & 1.879 & -0.353 & 1 \\
\hline Ti I & 5978.543 & 1.873 & -0.440 & 1 \\
\hline Ti I & 6064.629 & 1.046 & -1.888 & 1 \\
\hline Ti I & 6126.217 & 1.066 & -1.369 & 1 \\
\hline Fe I & 5322.041 & 2.279 & -2.840 & 2,3 \\
\hline $\mathrm{Fe} \mathrm{I}$ & 5811.919 & 4.143 & -2.430 & 2,3 \\
\hline $\mathrm{Fe} \mathrm{I}$ & 5853.161 & 1.485 & -5.280 & 2,3 \\
\hline $\mathrm{Fe} \mathrm{I}$ & 5855.086 & 4.608 & -1.600 & 2,3 \\
\hline $\mathrm{Fe} \mathrm{I}$ & 5856.096 & 4.295 & -1.640 & 2,3 \\
\hline Fe I & 5858.785 & 4.221 & -2.260 & 2,3 \\
\hline Fe I & 5927.797 & 4.652 & -1.090 & 2,3 \\
\hline Fe I & 5933.803 & 4.639 & -2.230 & 2,3 \\
\hline Fe I & 5940.997 & 4.178 & -2.150 & 2,3 \\
\hline Fe I & 5956.706 & 0.859 & -4.605 & 2,3 \\
\hline Fe I & 5969.578 & 4.283 & -2.730 & 2,3 \\
\hline Fe I & 6019.364 & 3.573 & -3.360 & 2,3 \\
\hline Fe I & 6027.051 & 4.076 & -1.090 & 2,3 \\
\hline Fe I & 6054.080 & 4.372 & -2.310 & 2,3 \\
\hline Fe I & 6105.130 & 4.549 & -2.050 & 2,3 \\
\hline Fe I & 6151.618 & 2.176 & -3.290 & 2,3 \\
\hline Fe I & 6157.728 & 4.076 & -1.110 & 2,3 \\
\hline $\mathrm{Fe} \mathrm{I}$ & 6159.380 & 4.608 & -1.970 & 2,3 \\
\hline $\mathrm{Fe} \mathrm{I}$ & 6165.360 & 4.142 & -1.470 & 2,3 \\
\hline Fe I & 6173.336 & 2.223 & -2.880 & 2,3 \\
\hline Fe II & 4491.407 & 2.855 & -2.490 & 2,3 \\
\hline Fe II & 4508.290 & 2.856 & -2.310 & 2,3 \\
\hline Fe II & 4620.520 & 2.828 & -3.230 & 2,3 \\
\hline Fe II & 5197.559 & 3.230 & -2.250 & 2,3 \\
\hline Fe II & 5264.810 & 3.231 & -3.150 & 2,3 \\
\hline Fe II & 5325.559 & 3.221 & -3.170 & 2,3 \\
\hline Fe II & 5414.046 & 3.221 & -3.620 & 2,3 \\
\hline Fe II & 5425.247 & 3.199 & -3.210 & 2,3 \\
\hline Fe II & 6149.246 & 3.889 & -2.720 & 2,3 \\
\hline $\mathrm{Zn} \mathrm{I}$ & 4722.153 & 4.030 & -0.338 & 2 \\
\hline $\mathrm{Zn} \mathrm{I}$ & 4810.528 & 4.078 & -0.137 & 2 \\
\hline
\end{tabular}

References.- (1) Provided by E. Luck (2003, private communication)- compilation from various sources.

(2) From Kurucz Atomic Line Database (Kurucz \& Bell 1995). (3) $\log g f$-values derived from our solar spectrum and $\chi$ from Kurucz \& Bell (1995). 
TABLE 2

Stellar Parameters

\begin{tabular}{|c|c|c|c|c|c|c|c|}
\hline HD & Other Name & B-V (1) & $\mathrm{T}_{e f f}(\mathrm{~K})$ & $\log g\left(\mathrm{~cm} \mathrm{~s}^{-2}\right)$ & $\xi\left(\mathrm{km} \mathrm{s}^{-1}\right)$ & $\zeta\left(\mathrm{km} \mathrm{s}^{-1}\right)(2)$ & $v \sin i\left(\mathrm{~km} \mathrm{~s}^{-1}\right)$ \\
\hline 26784 & vB 19 & 0.51 & 6450 & 4.2 & 1.1 & 4.89 & 15.7 \\
\hline 27808 & vB 48 & 0.52 & 6400 & 4.3 & 1.0 & 4.86 & 11.0 \\
\hline 30809 & vB 143 & 0.53 & 6400 & 4.2 & 0.9 & 4.79 & 10.1 \\
\hline 28205 & vB 65 & 0.54 & 6250 & 4.3 & 1.0 & 4.71 & 8.8 \\
\hline 28635 & vB 88 & 0.54 & 6250 & 4.3 & 0.8 & 4.69 & 1.0 \\
\hline 26257 & HIP 19386 & 0.55 & 6300 & 4.3 & 1.0 & 4.59 & 5.9 \\
\hline 35768 & HIP 25639 & 0.56 & 6300 & 4.3 & 1.0 & 4.57 & 4.4 \\
\hline 27406 & vB 31 & 0.56 & 6200 & 4.3 & 1.0 & 4.54 & 10.0 \\
\hline 28237 & vB 66 & 0.56 & 6250 & 4.3 & 0.7 & 4.54 & 8.6 \\
\hline 20430 & vB 1 & 0.57 & 6250 & 4.4 & 0.8 & 4.49 & 5.5 \\
\hline 29419 & vB 105 & 0.58 & 6100 & 4.4 & 0.8 & 4.42 & 2.5 \\
\hline 30589 & vB 118 & 0.58 & 6100 & 4.4 & 0.8 & 4.40 & 5.3 \\
\hline 27835 & vB 49 & 0.59 & 6050 & 4.4 & 0.8 & 4.31 & 2.8 \\
\hline 25825 & vB 10 & 0.59 & 6100 & 4.5 & 0.7 & 4.29 & 6.2 \\
\hline 27859 & vB 52 & 0.60 & 6050 & 4.4 & 0.5 & 4.24 & 6.5 \\
\hline 28344 & vB 73 & 0.61 & 6000 & 4.4 & 0.6 & 4.17 & 6.8 \\
\hline 20439 & vB 2 & 0.62 & 6050 & 4.4 & 0.6 & 4.11 & 5.5 \\
\hline 28992 & vB 97 & 0.63 & 5900 & 4.4 & 0.8 & 4.00 & 5.4 \\
\hline 26767 & vB 18 & 0.64 & 5900 & 4.4 & 0.8 & 3.94 & 5.4 \\
\hline 26736 & vB 15 & 0.66 & 5750 & 4.4 & 0.7 & 3.80 & 5.4 \\
\hline 28099 & vB 64 & 0.66 & 5800 & 4.4 & 0.7 & 3.75 & 3.4 \\
\hline \multirow[t]{2}{*}{26756} & vB 17 & 0.69 & 5650 & 4.5 & 0.8 & 3.53 & 4.5 \\
\hline & HIP 13600 & 0.70 & 5600 & 4.5 & 0.6 & 3.44 & 1.8 \\
\hline 27282 & vB 27 & 0.72 & 5600 & 4.5 & 0.7 & 3.32 & 4.9 \\
\hline 240648 & HIP 23750 & 0.73 & 5600 & 4.5 & 0.7 & 3.25 & 4.9 \\
\hline 19902 & HIP 14976 & 0.73 & 5600 & 4.5 & 0.8 & 3.23 & 1.5 \\
\hline 28593 & vB 87 & 0.73 & 5550 & 4.5 & 0.8 & 3.22 & 4.0 \\
\hline 31609 & vB 127 & 0.74 & 5550 & 4.5 & 0.6 & 3.19 & 2.5 \\
\hline 26015B & vB 12 & 0.74 & 5250 & 4.5 & 0.8 & 3.17 & 4.5 \\
\hline 28805 & vB 92 & 0.74 & 5500 & 4.5 & 0.7 & 3.17 & 3.8 \\
\hline 27250 & vB 26 & 0.75 & 5550 & 4.5 & 0.8 & 3.13 & 3.5 \\
\hline 27732 & vB 42 & 0.76 & 5500 & 4.5 & 0.8 & 3.03 & 3.8 \\
\hline 32347 & vB 187 & 0.77 & 5500 & 4.5 & 0.8 & 2.98 & 4.4 \\
\hline 242780 & HIP 24923 & 0.77 & 5500 & 4.5 & 0.7 & 2.98 & 4.8 \\
\hline 283704 & vB 76 & 0.77 & 5500 & 4.5 & 0.8 & 2.97 & 2.5 \\
\hline 284574 & vB 109 & 0.81 & 5350 & 4.5 & 0.8 & 2.63 & 4.6 \\
\hline 284253 & vB 21 & 0.81 & 5350 & 4.5 & 0.5 & 2.62 & 2.3 \\
\hline 285773 & vB 79 & 0.83 & 5300 & 4.5 & 0.5 & 2.48 & 3.4 \\
\hline 30505 & vB 116 & 0.83 & 5300 & 4.6 & 0.8 & 2.46 & 3.8 \\
\hline \multirow[t]{3}{*}{28258} & vB 178 & 0.84 & 5350 & 4.6 & 0.7 & 2.42 & 3.5 \\
\hline & vB 4 & 0.84 & 5250 & 4.6 & 0.6 & 2.38 & 2.8 \\
\hline & vB 153 & 0.86 & 5200 & 4.6 & 0.7 & 2.30 & 3.8 \\
\hline 27771 & vB 46 & 0.86 & 5200 & 4.6 & 0.8 & 2.30 & 3.0 \\
\hline 28462 & vB 180 & 0.87 & 5250 & 4.6 & 1.0 & 2.22 & 3.9 \\
\hline 29159 & vB 99 & 0.87 & 5000 & 4.6 & 0.5 & 2.18 & 3.4 \\
\hline 28878 & vB 93 & 0.89 & 5150 & 4.6 & 0.7 & 2.03 & 3.8 \\
\hline 285367 & HIP 19098 & 0.89 & 5150 & 4.6 & 0.8 & 2.03 & 3.7 \\
\hline \multirow[t]{2}{*}{285252} & vB 7 & 0.90 & 5050 & 4.6 & 0.8 & 1.99 & 3.8 \\
\hline & vB 5 & 0.92 & 5050 & 4.6 & 0.7 & 1.83 & 1.9 \\
\hline 28977 & vB 183 & 0.92 & 5150 & 4.6 & 0.9 & 1.80 & 3.5 \\
\hline 18632 & HIP 13976 & 0.93 & 5000 & 4.6 & 0.7 & 1.76 & 2.9 \\
\hline \multirow[t]{2}{*}{285830} & vB 179 & 0.93 & 5050 & 4.6 & 0.6 & 1.73 & 3.6 \\
\hline & HIP 23312 & 0.96 & 5100 & 4.6 & 0.7 & 1.52 & 2.4 \\
\hline 285690 & vB 25 & 0.98 & 4900 & 4.6 & 0.8 & 1.35 & 2.5 \\
\hline 14127 & HIP 10672 & 0.57 & 6200 & 4.4 & 0.8 & 4.49 & 6.3 \\
\hline
\end{tabular}

${ }^{1} \mathrm{~B}-\mathrm{V}$ values taken from Allende Prieto \& Lambert (1999)

${ }^{2}$ Macroturbulence derived from Saar \& Osten (1997) 
TABLE 3

Elemental Abundances

\begin{tabular}{|c|c|c|c|c|c|c|c|c|}
\hline HD & Other Name & {$[\mathrm{Fe} / \mathrm{H}]$} & {$[\mathrm{Na} / \mathrm{Fe}]$} & {$[\mathrm{Mg} / \mathrm{Fe}]$} & {$[\mathrm{Si} / \mathrm{Fe}]$} & {$[\mathrm{Ca} / \mathrm{Fe}]$} & {$[\mathrm{Ti} / \mathrm{Fe}]$} & {$[\mathrm{Zn} / \mathrm{Fe}]$} \\
\hline 26784 & vB 19 & 0.23 & 0.07 & -0.08 & -0.01 & 0.08 & 0.08 & -0.19 \\
\hline 27808 & vB 48 & 0.15 & 0.07 & 0.00 & 0.06 & 0.15 & 0.15 & 0.11 \\
\hline 30809 & vB 143 & 0.19 & 0.10 & -0.06 & 0.02 & 0.29 & 0.15 & -0.14 \\
\hline 28205 & vB 65 & 0.10 & 0.03 & -0.08 & 0.08 & 0.17 & 0.10 & -0.05 \\
\hline 28635 & vB 88 & 0.09 & 0.05 & -0.05 & 0.05 & 0.11 & 0.01 & -0.09 \\
\hline 26257 & HIP 19386 & 0.11 & 0.00 & -0.11 & 0.09 & 0.12 & 0.03 & -0.03 \\
\hline 35768 & HIP 25639 & 0.08 & 0.08 & -0.02 & 0.05 & 0.12 & 0.12 & -0.10 \\
\hline 27406 & vB 31 & 0.15 & 0.05 & -0.06 & 0.01 & 0.19 & 0.07 & -0.06 \\
\hline 28237 & $\mathrm{vB} 66$ & 0.14 & 0.04 & -0.01 & 0.02 & 0.22 & 0.03 & 0.04 \\
\hline 20430 & $\mathrm{vB} 1$ & 0.30 & 0.02 & -0.05 & 0.00 & 0.16 & 0.07 & -0.11 \\
\hline 29419 & vB 105 & 0.13 & -0.06 & -0.05 & 0.01 & 0.11 & -0.04 & -0.07 \\
\hline 30589 & vB 118 & 0.15 & 0.00 & -0.08 & 0.02 & 0.09 & 0.00 & -0.12 \\
\hline 27835 & vB 49 & 0.09 & 0.00 & -0.06 & 0.05 & 0.10 & 0.03 & -0.08 \\
\hline 25825 & vB 10 & 0.15 & 0.02 & -0.07 & -0.04 & 0.12 & -0.03 & -0.01 \\
\hline 27859 & vB 52 & 0.14 & 0.01 & -0.05 & 0.00 & 0.13 & 0.03 & 0.02 \\
\hline 28344 & vB 73 & 0.18 & 0.01 & -0.09 & -0.03 & 0.11 & 0.01 & 0.00 \\
\hline 20439 & vB 2 & 0.30 & 0.01 & -0.06 & -0.02 & 0.13 & 0.04 & -0.03 \\
\hline 28992 & vB 97 & 0.12 & -0.01 & -0.11 & 0.02 & 0.09 & 0.03 & -0.05 \\
\hline 26767 & vB 18 & 0.12 & -0.01 & -0.01 & 0.04 & 0.13 & 0.05 & 0.00 \\
\hline 26736 & vB 15 & 0.09 & 0.05 & 0.01 & 0.06 & 0.14 & 0.00 & 0.08 \\
\hline 28099 & vB 64 & 0.10 & 0.02 & -0.04 & 0.05 & 0.11 & 0.04 & -0.04 \\
\hline \multirow[t]{2}{*}{26756} & vB 17 & 0.06 & 0.01 & -0.03 & 0.08 & 0.08 & 0.02 & -0.08 \\
\hline & HIP 13600 & 0.02 & 0.03 & 0.02 & 0.04 & 0.09 & 0.03 & -0.04 \\
\hline 27282 & vB 27 & 0.15 & 0.01 & -0.01 & 0.03 & 0.08 & 0.05 & -0.03 \\
\hline 240648 & HIP 23750 & 0.15 & -0.01 & -0.05 & 0.02 & 0.09 & 0.03 & -0.02 \\
\hline 19902 & HIP 14976 & 0.09 & 0.00 & -0.06 & 0.07 & 0.05 & 0.03 & -0.08 \\
\hline 28593 & vB 87 & 0.11 & 0.02 & -0.07 & 0.06 & 0.03 & 0.02 & -0.08 \\
\hline 31609 & vB 127 & 0.15 & 0.01 & -0.08 & 0.00 & 0.02 & -0.01 & -0.10 \\
\hline 26015B & vB 12 & 0.17 & -0.04 & -0.07 & 0.00 & -0.01 & -0.01 & -0.09 \\
\hline 28805 & vB 92 & 0.08 & -0.04 & -0.05 & 0.06 & 0.06 & -0.04 & -0.02 \\
\hline 27250 & vB 26 & 0.09 & -0.02 & 0.00 & 0.09 & 0.04 & 0.06 & -0.09 \\
\hline 27732 & vB 42 & 0.10 & -0.05 & -0.04 & 0.06 & 0.08 & -0.04 & -0.06 \\
\hline 32347 & vB 187 & 0.11 & -0.05 & -0.02 & 0.06 & 0.09 & 0.03 & -0.05 \\
\hline 242780 & HIP 24923 & 0.12 & 0.02 & -0.05 & 0.07 & 0.09 & 0.07 & -0.04 \\
\hline 283704 & vB 76 & 0.09 & 0.01 & -0.04 & 0.07 & 0.06 & 0.03 & -0.07 \\
\hline 284574 & vB 109 & 0.13 & 0.01 & -0.04 & 0.08 & 0.08 & 0.05 & 0.00 \\
\hline 284253 & vB 21 & 0.14 & 0.02 & -0.06 & 0.00 & 0.06 & 0.02 & -0.05 \\
\hline 285773 & vB 79 & 0.14 & 0.01 & -0.09 & 0.02 & 0.02 & 0.00 & -0.06 \\
\hline 30505 & vB 116 & 0.13 & -0.05 & -0.09 & 0.05 & 0.02 & 0.03 & -0.07 \\
\hline \multirow[t]{3}{*}{28258} & vB 178 & 0.15 & -0.03 & -0.06 & 0.02 & 0.05 & -0.02 & -0.09 \\
\hline & $\mathrm{vB} 4$ & 0.15 & -0.02 & -0.09 & 0.02 & 0.02 & 0.03 & -0.07 \\
\hline & vB 153 & 0.10 & -0.03 & -0.04 & 0.07 & 0.06 & 0.07 & -0.01 \\
\hline 27771 & vB 46 & 0.08 & 0.00 & -0.07 & 0.14 & 0.00 & 0.05 & -0.01 \\
\hline 28462 & vB 180 & 0.08 & -0.02 & -0.02 & 0.09 & 0.01 & 0.03 & -0.08 \\
\hline 29159 & vB 99 & 0.09 & 0.04 & -0.13 & 0.17 & -0.12 & 0.05 & 0.03 \\
\hline 28878 & vB 93 & 0.13 & -0.05 & -0.09 & 0.06 & 0.01 & 0.01 & -0.05 \\
\hline 285367 & HIP 19098 & 0.11 & -0.05 & -0.08 & 0.05 & 0.03 & 0.02 & -0.10 \\
\hline \multirow[t]{2}{*}{285252} & vB 7 & 0.15 & -0.03 & -0.11 & 0.10 & -0.08 & -0.06 & -0.07 \\
\hline & vB 5 & 0.16 & -0.08 & -0.12 & 0.04 & -0.05 & -0.01 & -0.13 \\
\hline 28977 & vB 183 & 0.13 & -0.01 & -0.09 & 0.03 & 0.03 & 0.07 & -0.14 \\
\hline 18632 & HIP 13976 & 0.18 & -0.10 & -0.10 & 0.06 & -0.02 & 0.03 & -0.15 \\
\hline \multirow[t]{2}{*}{285830} & vB 179 & 0.22 & -0.08 & -0.15 & 0.03 & -0.03 & 0.03 & -0.12 \\
\hline & HIP 23312 & 0.18 & -0.04 & -0.11 & -0.04 & 0.00 & 0.09 & -0.20 \\
\hline 285690 & vB 25 & 0.08 & -0.08 & -0.06 & 0.20 & -0.03 & 0.02 & -0.09 \\
\hline 14127 & HIP 10672 & -0.12 & -0.09 & -0.01 & 0.02 & 0.14 & 0.10 & -0.05 \\
\hline
\end{tabular}


TABLE 4

Comparison of Derived Parameters for Stars Common to Both Samples

\begin{tabular}{cccccc}
\hline \hline Star & $\Delta[\mathrm{Fe} / \mathrm{H}]$ & $\Delta T_{\text {eff }}(\mathrm{K})$ & $\Delta \log g$ & $\Delta \xi$ & $\left(\mathrm{km} \mathrm{s}^{-1}\right)$ \\
\hline vB 19 & 0.06 & 0 & 0 & 0.1 \\
vB 10 & 0.03 & 0 & 0 & 0 \\
vB 73 & 0.04 & 0 & 0 & 0 \\
vB 118 & 0.06 & 50 & 0 & 0 \\
vB 105 & 0.08 & 0 & -0.1 & -0.1 \\
vB 66 & 0.03 & 0 & 0 & 0 \\
vB 88 & 0.07 & 50 & 0 & -0.1 \\
vB 65 & 0.09 & 0 & 0 & -0.1 \\
vB 143 & 0.06 & 0 & -0.1 & -0.1 \\
vB 48 & 0.01 & 0 & 0 & 0 \\
vB 31 & 0.07 & 0 & 0 & -0.1 \\
\hline
\end{tabular}


TABle 5

Differential Abundances

\begin{tabular}{|c|c|c|c|c|c|c|c|c|}
\hline HD & Other Name & $\Delta[\mathrm{Fe} / \mathrm{H}]$ & $\Delta[\mathrm{Na} / \mathrm{Fe}]$ & $\Delta[\mathrm{Mg} / \mathrm{Fe}]$ & $\Delta[\mathrm{Si} / \mathrm{Fe}]$ & $\Delta[\mathrm{Ca} / \mathrm{Fe}]$ & $\Delta[\mathrm{Ti} / \mathrm{Fe}]$ & $\Delta[\mathrm{Zn} / \mathrm{Fe}]$ \\
\hline 26784 & vB 19 & 0.15 & 0.08 & 0.02 & -0.02 & 0.03 & 0.12 & 0.01 \\
\hline 27808 & vB 48 & 0.07 & 0.08 & 0.02 & -0.01 & 0.16 & 0.04 & 0.10 \\
\hline 30809 & vB 143 & 0.11 & 0.01 & -0.04 & -0.06 & 0.16 & 0.00 & -0.14 \\
\hline 28205 & vB 65 & 0.01 & 0.04 & -0.05 & 0.02 & 0.11 & 0.03 & -0.04 \\
\hline 28635 & vB 88 & 0.05 & -0.06 & -0.07 & -0.05 & -0.02 & -0.12 & -0.13 \\
\hline 26257 & HIP 19386 & 0.02 & 0.07 & -0.08 & 0.03 & 0.06 & -0.04 & -0.03 \\
\hline 35768 & HIP 25639 & 0.00 & 0.03 & 0.00 & -0.02 & 0.03 & 0.09 & -0.10 \\
\hline 27406 & vB 31 & 0.07 & 0.03 & -0.04 & -0.05 & 0.11 & -0.03 & -0.06 \\
\hline 28237 & vB 66 & 0.07 & -0.01 & 0.01 & -0.06 & 0.14 & -0.07 & 0.03 \\
\hline 20430 & vB 1 & 0.21 & -0.08 & -0.04 & -0.09 & 0.08 & 0.00 & -0.11 \\
\hline 29419 & vB 105 & 0.04 & 0.39 & -0.03 & -0.04 & 0.02 & -0.09 & -0.09 \\
\hline 30589 & vB 118 & 0.07 & -0.03 & -0.07 & -0.05 & 0.01 & -0.08 & 0.09 \\
\hline 27835 & vB 49 & 0.02 & -0.01 & -0.05 & -0.02 & 0.01 & -0.06 & -0.09 \\
\hline 25825 & vB 10 & 0.08 & -0.02 & -0.06 & -0.14 & 0.03 & -0.13 & -0.02 \\
\hline 27859 & vB 52 & 0.06 & -0.01 & -0.03 & -0.07 & 0.05 & -0.06 & 0.02 \\
\hline 28344 & vB 73 & 0.09 & 0.00 & -0.06 & -0.09 & 0.04 & -0.09 & 0.00 \\
\hline 20439 & vB 2 & 0.20 & -0.08 & -0.02 & -0.09 & 0.07 & -0.03 & -0.02 \\
\hline 28992 & vB 97 & 0.04 & -0.03 & -0.09 & -0.05 & 0.01 & -0.05 & -0.06 \\
\hline 26767 & vB 18 & 0.03 & 0.04 & 0.02 & -0.03 & 0.07 & -0.03 & 0.01 \\
\hline 26736 & vB 15 & -0.01 & 0.03 & 0.05 & 0.00 & 0.08 & -0.07 & 0.00 \\
\hline 28099 & vB 64 & 0.02 & -0.01 & -0.02 & -0.03 & 0.03 & -0.04 & 0.10 \\
\hline \multirow[t]{2}{*}{26756} & vB 17 & -0.03 & 0.02 & 0.00 & 0.01 & 0.01 & -0.06 & -0.08 \\
\hline & HIP 13600 & -0.07 & 0.00 & 0.05 & -0.03 & 0.02 & -0.05 & -0.03 \\
\hline 27282 & vB 27 & 0.05 & 0.00 & 0.03 & -0.03 & 0.02 & -0.02 & -0.01 \\
\hline 240648 & HIP 23750 & 0.08 & -0.03 & -0.04 & -0.08 & 0.01 & -0.08 & -0.04 \\
\hline 19902 & HIP 14976 & 0.01 & 0.01 & -0.04 & -0.02 & -0.02 & -0.06 & -0.08 \\
\hline 28593 & vB 87 & 0.02 & 0.01 & -0.04 & -0.01 & -0.03 & -0.05 & -0.08 \\
\hline 31609 & vB 127 & 0.06 & -0.04 & -0.05 & -0.07 & -0.05 & -0.09 & -0.09 \\
\hline $26015 \mathrm{~B}$ & vB 12 & 0.07 & -0.04 & -0.03 & -0.06 & -0.07 & -0.08 & -0.07 \\
\hline 28805 & vB 92 & -0.03 & -0.01 & 0.00 & 0.00 & 0.01 & -0.09 & 0.00 \\
\hline 27250 & vB 26 & 0.01 & 0.32 & -0.01 & -0.03 & -0.07 & -0.09 & -0.09 \\
\hline 27732 & vB 42 & -0.03 & -0.01 & 0.00 & 0.01 & 0.01 & -0.02 & -0.06 \\
\hline 32347 & vB 187 & 0.01 & 0.02 & 0.02 & -0.02 & 0.03 & -0.12 & -0.04 \\
\hline 242780 & HIP 24923 & 0.03 & 0.00 & -0.04 & -0.01 & 0.01 & 0.00 & -0.06 \\
\hline 283704 & vB 76 & -0.01 & 0.01 & 0.00 & 0.00 & 0.00 & -0.05 & -0.05 \\
\hline 284574 & vB 109 & 0.06 & -0.01 & -0.03 & -0.02 & -0.01 & -0.05 & -0.02 \\
\hline 284253 & vB 21 & 0.03 & 0.02 & -0.01 & -0.06 & 0.01 & -0.03 & -0.03 \\
\hline 285773 & vB 79 & 0.04 & -0.04 & -0.05 & -0.05 & -0.04 & -0.07 & -0.05 \\
\hline 30505 & vB 116 & 0.03 & -0.03 & -0.05 & -0.03 & -0.03 & -0.04 & -0.05 \\
\hline \multirow[t]{3}{*}{28258} & vB 178 & 0.04 & 0.00 & -0.01 & -0.03 & 0.00 & -0.08 & -0.06 \\
\hline & vB 4 & 0.05 & -0.03 & 0.04 & -0.05 & 0.04 & -0.05 & -0.05 \\
\hline & vB 153 & $\ldots$ & $\ldots$ & $\ldots$ & $\ldots$ & $\ldots$ & $\ldots$ & $\ldots$ \\
\hline 27771 & vB 46 & -0.02 & -0.02 & -0.03 & 0.08 & -0.05 & -0.01 & 0.01 \\
\hline 28462 & vB 180 & -0.02 & 0.04 & 0.02 & 0.02 & -0.04 & -0.06 & -0.06 \\
\hline 29159 & vB 99 & -0.02 & -0.14 & -0.08 & -0.01 & -0.17 & -0.01 & 0.06 \\
\hline 28878 & vB 93 & 0.02 & -0.04 & -0.04 & -0.01 & -0.04 & -0.04 & -0.03 \\
\hline 285367 & HIP 19098 & -0.01 & -0.04 & -0.02 & 0.00 & 0.00 & -0.03 & -0.06 \\
\hline \multirow[t]{2}{*}{285252} & vB 7 & 0.04 & -0.05 & -0.06 & 0.04 & -0.13 & -0.12 & -0.04 \\
\hline & vB 5 & 0.03 & -0.04 & -0.05 & -0.01 & -0.08 & -0.04 & -0.08 \\
\hline 28977 & vB 183 & 0.01 & 0.01 & -0.03 & -0.02 & -0.01 & 0.02 & -0.09 \\
\hline 18632 & HIP 13976 & 0.05 & -0.07 & -0.03 & 0.01 & -0.05 & -0.02 & -0.10 \\
\hline \multirow[t]{2}{*}{285830} & vB 179 & 0.10 & -0.06 & -0.09 & -0.03 & -0.07 & -0.02 & -0.08 \\
\hline & HIP 23312 & 0.05 & 0.00 & 0.05 & -0.08 & -0.03 & 0.04 & -0.14 \\
\hline 285690 & vB 25 & 0.02 & -0.12 & -0.06 & 0.08 & -0.12 & -0.09 & -0.10 \\
\hline 14127 & HIP 10672 & -0.20 & -0.02 & 0.01 & -0.04 & 0.07 & 0.00 & -0.04 \\
\hline
\end{tabular}


TABLE 6

Mean Cluster Abundances

\begin{tabular}{lccccccc}
\hline \hline & $\langle[\mathrm{Fe} / \mathrm{H}]\rangle$ & $\langle[\mathrm{Na} / \mathrm{Fe}]\rangle$ & $\langle[\mathrm{Mg} / \mathrm{Fe}]\rangle$ & $\langle[\mathrm{Si} / \mathrm{Fe}]\rangle$ & $\langle[\mathrm{Ca} / \mathrm{Fe}]\rangle$ & $\langle[\mathrm{Ti} / \mathrm{Fe}]\rangle$ & $\langle[\mathrm{Zn} / \mathrm{Fe}]\rangle$ \\
\hline Absolute & 0.13 & 0.01 & -0.06 & 0.05 & 0.07 & 0.03 & -0.06 \\
$\sigma$ & 0.05 & 0.09 & 0.04 & 0.05 & 0.07 & 0.05 & 0.06 \\
\hline Differential & 0.04 & -0.01 & -0.03 & -0.03 & 0.01 & -0.04 & -0.05 \\
$\sigma$ & 0.05 & 0.06 & 0.04 & 0.04 & 0.07 & 0.05 & 0.05 \\
\hline
\end{tabular}


TABLE 7

Abundance Dependencies on Model Parameters

\begin{tabular}{lcccccccc}
\hline \hline Example Star & Model Parameter & $\delta[\mathrm{Fe} / \mathrm{H}]$ & $\delta[\mathrm{Na} / \mathrm{Fe}]$ & $\delta[\mathrm{Mg} / \mathrm{Fe}]$ & $\delta[\mathrm{Si} / \mathrm{Fe}]$ & $\delta[\mathrm{Ca} / \mathrm{Fe}]$ & $\delta[\mathrm{Ti} / \mathrm{Fe}]$ & $\delta[\mathrm{Zn} / \mathrm{Fe}]$ \\
\hline vB 65 & $\mathrm{T}_{\text {eff }} \pm 50$ & \pm 0.04 & $\mp 0.02$ & \pm 0.01 & $\mp 0.02$ & $\mp 0.01$ & \pm 0.01 & $\mp 0.01$ \\
$\left(T_{\text {eff }}=6250\right)$ & $\log g \pm 0.20$ & \pm 0.04 & $\mp 0.01$ & $\mp 0.02$ & $\mp 0.01$ & $\mp 0.02$ & $\mp 0.01$ & 0.00 \\
& $\xi \pm 0.2$ & $\mp 0.03$ & \pm 0.02 & 0.00 & \pm 0.01 & $\mp 0.01$ & $\mp 0.02$ & $\mp 0.06$ \\
\hline vB 7 & $T_{\text {eff }} \pm 50$ & \pm 0.01 & \pm 0.03 & \pm 0.01 & $\mp 0.03$ & \pm 0.03 & \pm 0.05 & $\mp 0.03$ \\
$\left(T_{\text {eff }}=5050\right)$ & $\log g \pm 0.2$ & \pm 0.01 & $\mp 0.06$ & $\mp 0.07$ & \pm 0.01 & $\mp 0.09$ & $\mp 0.02$ & $\mp 0.01$ \\
& $\xi \pm 0.2$ & $\mp 0.04$ & \pm 0.02 & $\mp 0.01$ & \pm 0.02 & 0.00 & $\mp 0.02$ & $\mp 0.01$ \\
\hline
\end{tabular}

